(2) Open Access Full Text Article

REVIEW

\title{
Inotuzumab ozogamicin: a CD22 mAb-drug conjugate for adult relapsed or refractory B-cell precursor acute lymphoblastic leukemia
}

This article was published in the following Dove Press journal:

Drug Design, Development and Therapy

\author{
Ilana R Yurkiewicz' \\ Lori Muffly ${ }^{2}$ \\ Michaela Liedtke'
}

'Department of Medicine, Division of Hematology, Stanford University School of Medicine, Stanford, CA, USA; ${ }^{2}$ Department of Medicine,

Division of Blood and Marrow

Transplantation, Stanford University School of Medicine, Stanford, CA, USA

Correspondence: Michaela Liedtke Department of Medicine, Division of Hematology, Stanford University School of Medicine, Stanford, CA 94305, USA

Tel +l 6504986000

Fax +l 6507245203

Email mliedtke@stanford.edu

\begin{abstract}
Despite improved rates of remission and cure in newly diagnosed adult acute lymphoblastic leukemia (ALL), the prognosis for patients with relapsed or refractory disease remains poor and the 5-year overall survival rate after relapse is under $10 \%$. A recent paradigm shift has focused on the promise of targeted immunotherapy rather than standard chemotherapy, as ALL blast cells express a variety of antigens, and monoclonal antibodies may be developed to identify and destroy the leukemic cells. Inotuzumab ozogamicin is a CD22 monoclonal antibody conjugated to the cytotoxic antibiotic calicheamicin. CD22 expression is detected on leukemic blasts in over $90 \%$ of patients with ALL. Based on promising results from preclinical studies, inotuzumab ozogamicin was tested in Phase 1/2 and Phase 3 clinical trials and it demonstrated improved complete remission rates, progression-free survival and overall survival in relapsed or refractory adult ALL compared to standard therapy. Ongoing studies are evaluating the value of inotuzumab ozogamicin when given in combination with chemotherapy as part of upfront treatment. This review discusses the drug's biochemical properties and mechanism of action, preclinical research outcomes, clinical trial results, adverse events and toxicities, drug approval and ongoing investigations.
\end{abstract}

Keywords: acute lymphoblastic leukemia, relapsed/refractory, inotuzumab ozogamicin, monoclonal antibody-drug conjugate, CD22

\section{Introduction}

The foundation of current treatment for adults with acute lymphoblastic leukemia (ALL) is intensive, multi-agent chemotherapy, followed by either consolidation and prolonged maintenance chemotherapy or risk-adapted allogeneic hematopoietic stem cell transplantation (HCT) in first remission. The application of intensified chemotherapy regimens from pediatrics has improved outcomes for the younger adult population. A majority of adults with newly diagnosed B-cell ALL (B-ALL) achieve complete remission (CR), ${ }^{1}$ and long-term disease free survival rates are now $30 \%-40 \%$ with standard adult ALL protocols and 50\%-60\% with pediatric approaches. ${ }^{2-6}$ However, most patients with CR will ultimately relapse, and the 5-year overall survival (OS) rate after relapse is a disappointing 7\%. ${ }^{3}$ In addition, a subset of patients do not respond to initial chemotherapy and are considered to have refractory ALL. ${ }^{7} \mathrm{HCT}$ is the only potentially curative treatment for patients with relapsed or refractory ALL. However, standard salvage chemotherapy regimens are associated with low response rates and often significant toxicity, limiting the ability of many adults with relapsed or refractory ALL to proceed to HCT. 
Given the poor outcomes of patients with relapsed and refractory ALL, recent research efforts have turned to immunotherapy approaches that directly target antigens on ALL cells. These agents offer novel mechanisms of action in addition to a side effect profile distinct from chemotherapy. Blast cells in ALL express a variety of specific antigens such as CD19, CD20, CD22, CD33 and CD52, and monoclonal antibodies (MoAbs) are now being used to target these antigens. For instance, one of the most widely used MoAbs is rituximab, an anti-CD20 antibody, which has been shown to improve survival in newly diagnosed CD20-positive B-ALL when used in combination with chemotherapy. ${ }^{8}$ In addition to naked antibodies such as rituximab, MoAb strategies include bispecific T-cell engagers or antibody-drug conjugates or immunoconjugates; several novel MoAbs - including ofatumumab, obinutuzumab, epratuzumab, denintuzumab mafodotin and moxetumomab pasudotox - are currently under investigation in both preclinical and clinical trials to assess safety and efficacy. ${ }^{9-11}$

Inotuzumab ozogamicin is an MoAb directed against CD22 and linked to a cytoxic agent from the class of calicheamicins. In 2017, as a result of the positive Phase 3 clinical trial comparing inotuzumab ozogamicin to standard chemotherapy, the drug (under the trade name Besponsa) was approved by the European Commission and the US Food and Drug Administration (FDA) for the treatment of adults with relapsed or refractory CD22-positive B-ALL. ${ }^{12,13}$ Inotuzumab ozogamicin currently remains under investiga- tion in ongoing trials designed to assess its efficacy as part of various combination therapies, as well as in pediatric ALL. In this manuscript, we will review inotuzumab ozogamicin's mechanism of action and pharmacokinetics, describe the results of preclinical research and clinical trials, and discuss ongoing and future investigations incorporating this agent.

\section{Mechanism of action and pharmacokinetic properties}

Inotuzumab consists of a humanized IgG4 MoAb against CD22, while ozogamicin derives from calicheamicins, a class of potent antitumor antibiotics that target DNA in the minor groove and induce strand scission. The CD22 receptor is a highly suitable therapeutic target, as it is expressed on most B-cell malignancies such as B-cell ALL, chronic lymphocytic leukemia, non-Hodgkin lymphoma (NHL) and hairy cell leukemia, but not in normal tissues, including hematopoietic stem cells and hematopoietic precursors of B lymphocytes. ${ }^{14}$ While the function of the transmembrane sialoglycoprotein CD22 is not completely clear, it is thought to regulate B-cell migration, B-lymphocyte survival, signal transduction, B-cell homing and cellular adhesion. ${ }^{15}$

After the antibody portion of the compound binds to $\mathrm{CD} 22$ receptors on the surface of leukemic cells, the entire inotuzumab-calicheamicin conjugate enters the cell (Figure 1). Upon internalization, the antibody-drug conjugate is transported through endosomes and lysosomes where the

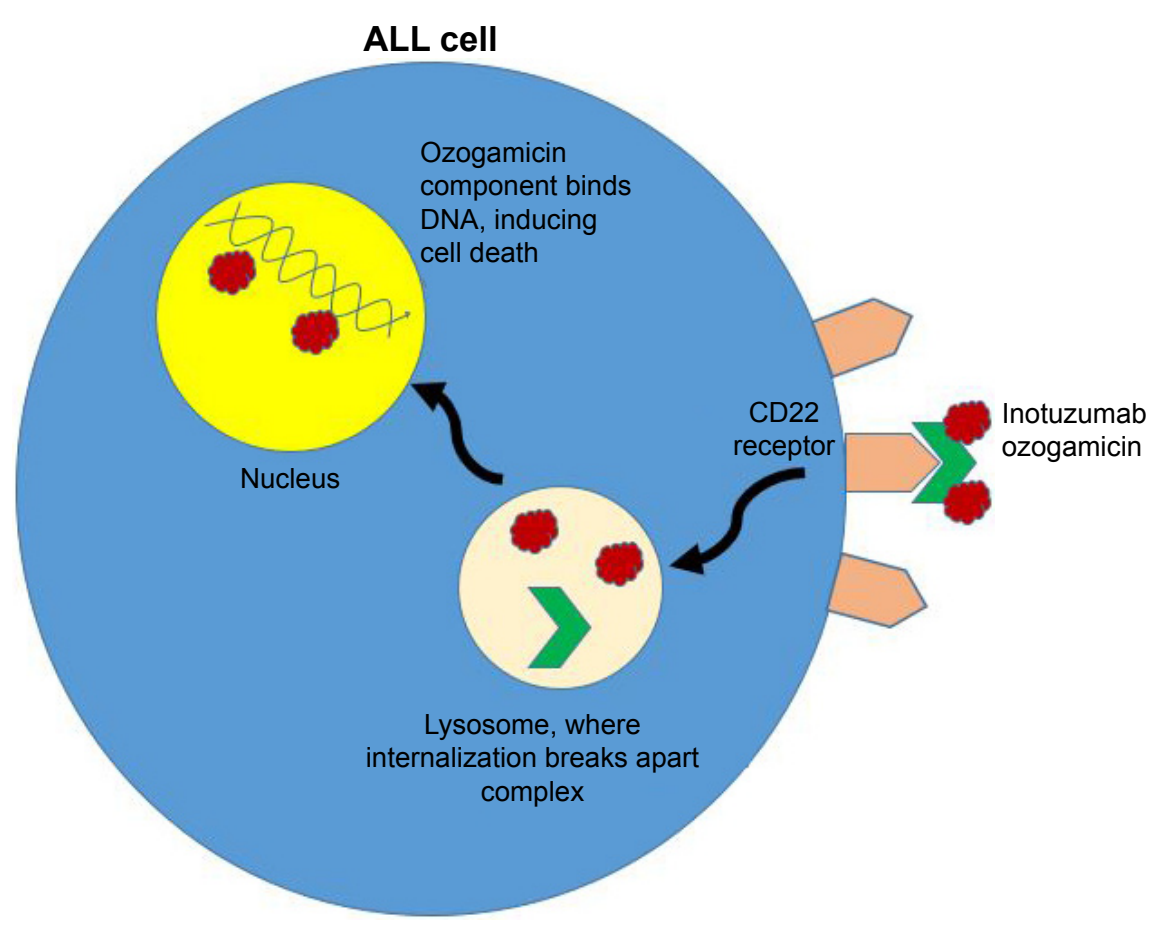

Figure I Depiction of inotuzumab ozogamicin's mechanism of action in tumor cells. Abbreviation: ALL, acute lymphoblastic leukemia. 
acidic environment hydrolyzes inotuzumab from the inactive ozogamicin. The latter component is then activated by intracellular glutathione and travels to the nucleus, where it induces cell cycle arrest and apoptosis, or cell death. Importantly, the effects of ozogamicin do not depend on cell cycle progression, meaning the molecule can induce apoptosis even in rapidly dividing cells. ${ }^{16-18}$ Another biochemical advantage is that compared to other B-lymphoid lineage surface antigens, CD22 is a better internalizing molecule. ${ }^{16,19}$

The pharmacokinetic properties of inotuzumab ozogamicin were analyzed in initial Phase 1 studies. In a Phase 1 trial in B-cell NHL, the pharmacokinetics of the drug demonstrated nonlinear disposition; there was increased drug exposure with either higher doses or increased number of doses of the drug. The mean end of infusion peak concentrations $\left(\mathrm{C}_{\max }\right)$ for 1.8 $\mathrm{mg} / \mathrm{m}^{2}$ once every 4 weeks and $2.4 \mathrm{mg} / \mathrm{m}^{2}$ once every 3 weeks could not be distinguished. No antibodies to the drug were detectable, except for one participant with a low baseline response. ${ }^{20}$ In another Phase 1 study of ten patients, drug exposure for inotuzumab ozogamicin and total calicheamicin $\left(\mathrm{C}_{\max }\right.$ and area under the concentration-time curve) increased with the number of doses. The $\mathrm{C}_{\text {max }}$ of inotuzumab ozogamicin was typically observed at termination or shortly after the infusion ended, while the $\mathrm{C}_{\max }$ of total calicheamicin was typically observed within 4 hours after starting inotuzumab ozogamicin. No antibodies to inotuzumab ozogamicin were detected during this study, either. ${ }^{21}$

\section{Preclinical investigation}

Much of the initial preclinical work with inotuzumab ozogamicin was done in relation to lymphoma. In mice, inotuzumab ozogamicin was effective against CD22 lymphoma xenografts, causing near-complete regression of both a Burkitt lymphoma cell line and an NHL cell line. In vitro studies demonstrated superiority compared to standard CHOP chemotherapy (consisting of cyclophosphamide, doxorubicin, vincristine and prednisolone) for the Burkitt lymphoma cell line. ${ }^{18,22}$ In additional studies, inotuzumab ozogamicin resulted in sustained regression in relapsed/refractory B-cell lymphoma xenografts previously treated with CHOP or CVP (consisting of cyclophosphamide, vincristine and prednisolone). ${ }^{23}$

As inotuzumab ozogamicin showed promising antitumor activity in preclinical NHL xenografts, it was explored both in vitro and in vivo in B-cell ALL models. In one in vitro study, inotuzumab ozogamicin demonstrated dose-dependent cellular destruction in a majority of cells derived from pediatric primary B-cell precursor ALL. ${ }^{24}$ Of note, the susceptibility of ALL cell lines to apoptosis induced by inotuzumab ozogamicin was found to be higher compared to NHL cell lines. In an in vivo investigation using B-cell ALL xenografts growing subcutaneously or as disseminated tumors, inotuzumab ozogamicin induced complete tumor regression and long-term survival of treated mice, while mice not treated with inotuzumab ozogamicin had disseminated disease by day 77 with an average survival of 5 days. ${ }^{25}$

The preclinical models have additionally shown that the combination of inotuzumab and ozogamicin has higher cytotoxic effects than ozogamicin alone, with an apparent dose-dependent response. In preclinical models of ALL and NHL, inotuzumab ozogamicin was 39 times more potent than unconjugated calicheamicin. ${ }^{16,19}$ Taken together, the results of these preclinical studies provided the basis for the development of inotuzumab ozogamicin as a potential therapeutic approach for patients with relapsed or refractory B-cell ALL.

\section{Clinical trials}

The first Phase 1 trial of inotuzumab ozogamicin was conducted in patients with relapsed or refractory CD22+ B-cell NHL. In this multicenter, open-label study, 79 patients received inotuzumab ozogamicin intravenously as a 1-hour infusion every 3-4 weeks. In the dose escalation part of the study, the maximum-tolerated dose (MTD) was determined to be $1.8 \mathrm{mg} / \mathrm{m}^{2}$, as at $2.4 \mathrm{mg} / \mathrm{m}^{2}$, two patients had doselimiting toxicities (grade 4 thrombocytopenia and grade 4 neutropenia). In Part 2, an expanded cohort received treatment at the MTD to determine preliminary efficacy, and the objective response rate at the end of treatment was 39\% for the 79 enrolled patients, $68 \%$ for all patients with follicular NHL treated at the MTD and 15\% for all patients with diffuse large B-cell lymphoma treated at the MTD. The median progression-free survival (PFS) was 317 days (95\% CI: 112-575 days). The main adverse event was thrombocytopenia (90\%), followed by asthenia (67\%), nausea (51\%) and neutropenia (51\%). While all cases of thrombocytopenia were considered clinically manageable (no major hemorrhages occurred, and the effect was reversible), the side effect prompted discontinuation of treatment in $24 \%$ of participants. Overall, the study concluded that inotuzumab ozogamicin demonstrated efficacy against CD22+ B-cell NHL with reversible thrombocytopenia as the main toxicity. ${ }^{20}$

Based on the promising preclinical data and early clinical results observed in NHL, several Phase 2 studies were performed to assess the safety and efficacy of inotuzumab ozogamicin in patients with relapsed or refractory CD22+ B-cell ALL (see Table 1 for a summary of key clinical trials using inotuzumab ozogamicin in relapsed or refractory adult B-cell ALL). A Phase 2 trial conducted at MD Anderson Cancer Center demonstrated that inotuzumab ozogamicin 
Table I Clinical trials of inotuzumab ozogamicin in relapsed or refractory adult B-cell ALL

\begin{tabular}{|c|c|c|c|c|c|}
\hline Phase & $\begin{array}{l}\text { Study } \\
\text { identifier }\end{array}$ & $\begin{array}{l}\text { Number of } \\
\text { patients }\end{array}$ & Dosing & CR/CRi (\%) & $\begin{array}{l}\text { Overall survival } \\
\text { (median in } \\
\text { months) }\end{array}$ \\
\hline 3 & NCTOI564784 & 109 & $1.8 \mathrm{mg} / \mathrm{m}^{2}$ per cycle $\left(0.8 \mathrm{mg} / \mathrm{m}^{2}\right.$ on day $\mathrm{I}, 0.5 \mathrm{mg} / \mathrm{m}^{2}$ on days 8 and $\left.\mathrm{I} 5\right)$ & 80.7 & 7.7 \\
\hline $1 / 2$ & NCT0I363297 & 72 & $1.8 \mathrm{mg} / \mathrm{m}^{2}$ per cycle $\left(0.8 \mathrm{mg} / \mathrm{m}^{2}\right.$ on day $\mathrm{I}, 0.5 \mathrm{mg} / \mathrm{m}^{2}$ on days 8 and $\left.\mathrm{I} 5\right)$ & 68 & 7.4 \\
\hline 2 & NCTOII 34575 & 49 & $1.8 \mathrm{mg} / \mathrm{m}^{2}$ per cycle & 58 & 6.2 \\
\hline 2 & NCT0I37I630 & 59 & $1.8 \mathrm{mg} / \mathrm{m}^{2}$ in cycle I, $1.3 \mathrm{mg} / \mathrm{m}^{2}$ in subsequent cycles & 59 & II \\
\hline
\end{tabular}

Abbreviations: ALL, acute lymphoblastic leukemia; CR, complete remission; CRi, complete remission with incomplete blood count recovery.

administered as monotherapy was highly active and safe in this patient population. In this study, 49 patients were treated with a single dose of inotuzumab ozogamicin of 1.3-1.8 $\mathrm{mg} / \mathrm{m}^{2}$ every 3-4 weeks, with subsequent doses given depending on the response and count recovery. ${ }^{26}$ In an attempt to reduce toxicity and maximize efficacy, the dosing schedule for a second cohort of 41 patients was changed to weekly infusions of inotuzumab ozogamicin dosed at $0.8 \mathrm{mg} / \mathrm{m}^{2}$ on day 1 and $0.5 \mathrm{mg} / \mathrm{m}^{2}$ on days 8 and 15 given every $3-4$ weeks. Combining the two cohorts, the overall patient population consisted of 90 patients with a median age of 39.5 years and included six children. Most patients received multiple prior regimens, including $11 \%$ who had undergone allogeneic HCT. CD22 positivity was at least $50 \%$ in all patients. In the combined cohort, an overall response rate (ORR) of 58\% was observed. Nineteen percent achieved complete response (CR), 30\% showed CR without platelet recovery and $9 \%$ showed marrow CR with incomplete blood count recovery (CRi). Results were similar when inotuzumab ozogamicin was administered as a single dose either on a monthly ( $57 \%$ ORR) or a weekly basis (59\% ORR). However, weekly dosing was better tolerated with lower rates of infusion-related fever, hypotension and hepatotoxicity. Median OS for the entire cohort was 6.2 months (1-year survival rate, $20 \%) .{ }^{27}$

An additional Phase 1/2 trial evaluated weekly dosing of inotuzumab ozogamicin for CD22-positive relapsed/ refractory B-cell ALL. ${ }^{28}$ In Phase 1 of this multisite trial, dose escalation determined the recommended Phase 2 dose to be $1.8 \mathrm{mg} / \mathrm{m}^{2}$ per cycle $\left(0.8 \mathrm{mg} / \mathrm{m}^{2}\right.$ on day $1,0.5 \mathrm{mg} / \mathrm{m}^{2}$ on days 8 and 15), with reduction to $1.6 \mathrm{mg} / \mathrm{m}^{2}$ per cycle after CR or CRi. Seventy-two adult patients with a median age of 45 years were treated, including 22\% with Philadelphia chromosome-positive $(\mathrm{Ph}+)$ ALL. All patients had received at least one prior salvage regimen, and $32 \%$ had undergone prior allogeneic HCT. In the overall population, $68 \%$ of participants achieved CR/CRi. Median PFS was 3.9 months, and median OS was 7.4 months (95\% CI: 5.7-9.2). Minimal residual disease (MRD) negativity was achieved in $84 \%$ of responding patients, with MRD-negative responses associated with higher drug exposure. The most common treatment-emergent adverse events were thrombocytopenia (36\%) and neutropenia (28\%) and the most common serious treatment-emergent adverse event was febrile neutropenia (22\%). Four patients experienced veno-occlusive disease/sinusoidal obstruction syndrome (VOD/SOS): two occurred during or shortly after study treatment without transplant, while the other two occurred post-study transplant. One patient died of VOD/SOS.

The sentinel INO-VATE trial was the first Phase 3 study to prove inotuzumab ozogamicin's superior efficacy compared to standard chemotherapy for adults with relapsed or refractory B-cell ALL, prompting its approval by national and international advisory groups. In this international, openlabel trial, adults with relapsed or refractory CD22-positive B-ALL were randomized to receive either inotuzumab ozogamicin or standard treatment with intensive chemotherapy consisting of either FLAG (fludarabine, cytosine arabinoside and granulocyte-colony stimulating factor) or high-dose cytosine arabinoside, or mitoxantrone and cytosine arabinoside. ${ }^{29}$ Inotuzumab ozogamicin was administered weekly at a dose of $0.8 \mathrm{mg} / \mathrm{m}^{2}$ on day 1 followed by $0.5 \mathrm{mg} / \mathrm{m}^{2}$ on days 8 and 15 repeated every 3-4 weeks. In patients who achieved $\mathrm{CR} / \mathrm{CRi}$, the subsequent dose on day 1 was reduced to $0.5 \mathrm{mg} / \mathrm{m}^{2}$. Patients who achieved CR could undergo HCT as per investigator's discretion.

Three hundred and twenty-six patients were enrolled, and analysis was based on intention to treat of the first 218 (109 in each group). No cross-over between the inotuzumab ozogamicin and standard treatment groups was permitted. The majority of patients in both arms of the study had received only one prior therapy, and the first remission duration was short ( $<12$ months) in 57\% of inotuzumab patients and $65 \%$ of chemotherapy patients. Prior allogeneic HCT had been performed in $16 \%$ and $20 \%$ of patients, respectively, in the inotuzumab ozogamicin and chemotherapy groups. CR rates were significantly higher in the inotuzumab ozogamicin group $(80.7 \%$ vs $29.4 \%$ in the standard therapy group, $P<0.001$ ), independent of age, first remission duration and salvage status. Among those with CR, significantly more 
patients in the inotuzumab ozogamicin arm demonstrated MRD negativity (78.4\% vs $28.1 \%, P<0.001)$. In addition, both PFS and OS were longer with inotuzumab ozogamicin: PFS was 5 vs 1.8 months $(P<0.001)$; median OS was 1 month longer (7.7 vs 6.7 months, hazard ratio: 0.77$)$. More patients in the inotuzumab ozogamicin group were able to proceed to post-study allogeneic HCT compared to the standard treatment group ( $41 \%$ vs $11 \%, P<0.001)$.

A recently published subgroup analysis compared the efficacy and safety of inotuzumab ozogamicin in younger (aged $<55$ years) and older patients enrolled in the INOVATE study. Remission rates, median duration of remission and PFS were comparable, but median OS was slightly shorter for patients aged $\geq 55$ years ( 5.6 vs 8.6 months, hazard ratio: 0.61 ). While there was no difference in discontinuation rate of drugs and adverse events were generally the same in both age groups, VOD/SOS was more commonly observed after HCT in older patients (41\% vs $17 \%) .{ }^{30}$

Based on the promising results of single-agent studies, inotuzumab ozogamicin has also been evaluated in combination with chemotherapy. In a Phase 2 study conducted at MD Anderson, 59 patients with relapsed/refractory CD22+ ALL received mini-hyper-CVD (cyclophosphamide and dexamethasone at 50\% dose reduction, no anthracycline, methotrexate at $75 \%$ dose reduction and cytarabine at $0.5 \mathrm{mg} / \mathrm{m}^{2}$ for four doses) and inotuzumab ozogamicin on day 3 of each cycle. The initial dose of inotuzumab ozogamicin was $1.8 \mathrm{mg} / \mathrm{m}^{2}$ in cycle 1 and $1.3 \mathrm{mg} / \mathrm{m}^{2}$ in subsequent cycles, but after observation of VOD/SOS, these doses were modified to 1.3 and $1 \mathrm{mg} / \mathrm{m}^{2}$, respectively. Rituximab was also given once per cycle to patients with CD20+ leukemia blasts. The ORR was $78 \%$, and $59 \%$ of patients achieved a CR with an MRD negativity rate of $87 \%$ in responders. The median relapse-free survival and OS were 8 and 11 months, respectively. Survival outcomes were superior for patients who received inotuzumab ozogamicin as the first salvage vs second or later salvage (median OS 17 vs 6 months). Of note, 26 patients $(44 \%)$ were able to proceed to HCT, supporting the role of inotuzumab ozogamicin as bridging therapy. The most commonly observed $\geq$ grade 3 adverse events were thrombocytopenia (81\%), infections (73\%) and hyperbilirubinemia (14\%). VOD/SOS was reported in nine patients $(15 \%){ }^{31}$

Of note, Phase 3 trial results for the molecule's efficacy in treating NHL have not been as promising. A Phase 3 trial was conducted after Phase $1 / 2$ trials demonstrated manageable safety and potential efficacy of inotuzumab ozogamicin as either monotherapy ${ }^{20,32}$ or in combination with rituximab. ${ }^{33,34}$ In 2013, the Phase 3 open-label, twoarm investigation compared inotuzumab ozogamicin to investigator's choice of bendamustine plus rituximab or gemcitabine plus rituximab for relapsed or refractory B-cell NHL. ${ }^{35}$ The trial was terminated early, as during interim evaluation, an independent Data Monitoring Committee concluded the trial would not meet its objective of showing improved OS. Similar toxicities were observed as in the ALL trial: specifically, two patients developed grade 3 VOD/SOS during inotuzumab ozogamicin treatment and one additional patient developed VOD/SOS 13 months after receiving inotuzumab ozogamicin and subsequent therapies including allogeneic HCT. No VOD/SOS events occurred in the control group.

\section{Toxicities and adverse events}

Based on the Phase 3 INO-VATE trial, inotuzumab ozogamicin was well tolerated overall with the exception of increased risk of VOD/SOS, discussed in more detail below. Overall, $86 \%$ of patients in the inotuzumab ozogamicin group had treatment-related adverse events, compared with $91 \%$ in the standard therapy group (no significant difference overall). The most common hematologic adverse events were cytopenias: neutropenia (36\% compared to $38 \%$ with standard therapy), thrombocytopenia ( $29 \%$ vs $48 \%$ ), anemia (18 vs 38\%) and leukopenia (17 vs 26\%). The most common non-hematological toxicities were febrile neutropenia ( 16 vs $43 \%$ with standard therapy), nausea (15 vs 34\%) and pyrexia (11 vs 25\%). ${ }^{19}$ Dose reduction due to adverse events was prompted in $2 \%$ of inotuzumab ozogamicin recipients (vs $1 \%$ of standard therapy patients). Drug discontinuation due to adverse events occurred in $9 \%$ of inotuzumab ozogamicin recipients (vs 5\% in the standard group). ${ }^{36}$ Results were similar in the Phase $1 / 2$ trial, in which cytopenias were the most common adverse events (thrombocytopenia at 36\% and neutropenia at $28 \%$ ). ${ }^{28}$

Notable differences in the INO-VATE trial between the treatment and control groups were cases of VOD/SOS, which were reported for up to 2 years after treatment. VOD/SOS occurred in 15 patients (11\%) who received inotuzumab ozogamicin, compared to one patient (1\%) who received standard therapy. Of the inotuzumab ozogamicin recipients who underwent HCT after the trial, ten patients (21\%) developed VOD/SOS after transplantation (compared to one patient, or $5 \%$, of the standard therapy recipients). ${ }^{29}$

Classically associated with HCT, hepatic VOD/SOS is characterized by hepatomegaly, right upper quadrant pain, weight gain and ascites. The disorder is thought to be caused 
by injury to the hepatic venous endothelium, as well as to the development of a prothrombotic state as a result of liver injury and coagulation factor disruption. Patients with severe VOD/SOS may develop multi-organ failure with resultant high risk of mortality. ${ }^{37}$ Risk factors for HCT-associated VOD/SOS include underlying liver disease, exposure to certain hepatotoxic medications or chemotherapeutic agents prior to HCT and the receipt of myeloablative HCT conditioning agents such as high-dose busulfan. VOD/SOS is a clinical diagnosis; the modified Seattle ${ }^{38}$ and Boston criteria ${ }^{39}$ are used to aid clinicians with the diagnosis. Historically, the management of VOD/SOS in the HCT setting has been largely supportive care, with focus on maintaining euvolemia, minimizing exposure to hepatotoxic agents, pain control and ascites management. Defibrotide, a pharmacological agent derived from porcine intestinal mucosal DNA, was recently approved by the US FDA for the treatment of HCT-associated VOD/SOS that is associated with additional kidney or lung abnormalities. Although the exact mechanism by which defibrotide treats VOD/SOS is not entirely clear, efficacy is likely linked to the purported anti-inflammatory and antithrombotic properties associated with defibrotide. ${ }^{40-42}$

Based on the available clinical trial data, VOD/SOS associated with inotuzumab is of greatest concern among those patients proceeding to HCT. Recently, an expert opinion panel was convened to develop consensus guidelines regarding inotuzumab and VOD/SOS risk. ${ }^{43}$ In general, and in keeping with the US FDA drug label for inotuzumab, expert recommendations include: limiting inotuzumab administration to two cycles in patients in whom allogeneic HCT is planned, avoiding HCT conditioning regimens containing two alkylating agents or thiotepa and avoiding concomitant hepatotoxic drugs whenever possible. Inotuzumab also comes with a Black Box warning contraindicating its use in patients with prior confirmed severe or serious ongoing VOD/SOS or with serious ongoing liver disease. ${ }^{12}$ The effectiveness of defibrotide to treat VOD/SOS developing following HCT in patients treated with pre-HCT inotuzumab has not been specifically reported.

\section{Ongoing investigations and future directions}

Inotuzumab ozogamicin is currently being investigated in multiple ongoing clinical trials evaluating its use as either monotherapy or as part of combination therapy in the upfront or relapsed/refractory setting. The Children's Oncology Group is conducting a study of inotuzumab ozogamicin in children and younger adults with relapsed/refractory B-cell ALL (NCT02981628). Inotuzumab ozogamicin is also being further studied in combination with chemotherapy in the front-line setting. The US Adult Intergroup is conducting a prospective study (A041501) evaluating the addition of inotuzumab ozogamicin to a pediatric-inspired chemotherapy backbone for patients aged 16-40 years with newly diagnosed CD22+ B-cell ALL (NCT03150693). Inotuzumab is also being evaluated as maintenance therapy following allogeneic HCT in a small Phase 1/2 study (NCT03104491).

In addition to inotuzumab ozogamicin, other immunotherapeutic approaches have proven successful in relapsed/ refractory ALL. Blinatumomab, a bispecific MoAb that directs CD3+ T cells to CD19+ leukemic blasts has also recently been approved for relapsed or refractory B-cell ALL. The agent is administered as a 24-hour infusion typically given over 4 weeks, and hospitalization is required when the infusion is initiated. In contrast, inotuzumab ozogamicin is administered as a 1-hour infusion and can be given in the outpatient setting. Blinatumomab also carries a unique set of risks. In the Phase 3 trial of 267 patients who received blinatumomab, $61.8 \%$ experienced a serious adverse event (the most common being neutropenia and infection, similar to inotuzumab), but 13 patients (4.9\%) experienced cytokine release syndrome (distinct from inotuzumab). ${ }^{44}$ Of note, when comparing the blinatumomab trial to the inotuzumab trial, medial survival was similar in both, and long-term disease control was not observed with either agent without HCT.

Beyond MoAbs, the other promising option for relapsed or refractory ALL is chimeric antigen receptor T cell (CAR-T) therapy, though this currently comes with a unique set of challenges. A CAR-T agent, tisagenlecleucel, was approved by the US FDA in August 2017 for pediatric patients aged 25 years or younger with relapsed or refractory B-cell ALL. For adults, a Phase 1 trial of 53 patients with relapsed B-ALL showed that a different CAR-T, 19-28z, resulted in a CR rate of $83 \%$ and a median survival of 12.9 months. However, 14 patients $(26 \%)$ developed severe cytokine release syndrome and $44 \%$ developed grade 2 or above neurotoxic effects. ${ }^{45}$ In addition to side effects, cost remains a limiting factor, with tisagenlecleucel carrying a price tag of $\$ 475,000 .{ }^{46}$ This compares to $\sim \$ 168,000$ for the duration of treatment with inotuzumab ozogamicin prior to purchaser discounts. ${ }^{47}$

\section{Conclusion}

Inotuzumab ozogamicin, a CD22 MoAb drug conjugate, has demonstrated overall safety and efficacy for adults with 
relapsed and refractory B-ALL based on preclinical studies and Phase 1, 2 and 3 clinical trials. Inotuzumab ozogamicin has been shown to improve CR rates, PFS and OS in relapsed or refractory adult B-cell ALL relative to standard treatment. While the most common adverse events are cytopenias and infections, the drug is associated with an increased risk of VOD/SOS. Based on promising results, the drug is now approved in the US and many countries in Europe, and ongoing investigations are exploring its role as either monotherapy or as part of combination therapy for various patient populations with B-cell ALL.

\section{Disclosure}

ML has received consulting fees and research support from Pfizer. The other authors report no conflicts of interest in this work.

\section{References}

1. Goldstone AH, Richards SM, Lazarus HM, et al. In adults with standardrisk acute lymphoblastic leukemia, the greatest benefit is achieved from a matched sibling allogeneic transplantation in first complete remission, and an autologous transplantation is less effective than conventional consolidation/maintenance chemotherapy in all patients: final results of the International ALL Trial (MRC UKALL XII/ECOG E2993). Blood. 2008;111(4):1827-1833.

2. Rowe JM, Buck G, Burnett AK, et al. Induction therapy for adults with acute lymphoblastic leukemia: results of more than 1500 patients from the international ALL trial: MRC UKALL XII/ECOG E2993. Blood. 2005;106(12):3760-3767.

3. Fielding AK, Richards SM, Chopra R, et al. Outcome of 609 adults after relapse of acute lymphoblastic leukemia (ALL); an MRC UKALL12/ ECOG 2993 study. Blood. 2007;109(3):944-950.

4. Deangelo DJ, Stevenson KE, Dahlberg SE, et al. Long-term outcome of a pediatric-inspired regimen used for adults aged 18-50 years with newly diagnosed acute lymphoblastic leukemia. Leukemia. 2015; 29(3):526-534.

5. Huguet F, Leguay T, Raffoux E, et al. Pediatric-inspired therapy in adults with Philadelphia chromosome-negative acute lymphoblastic leukemia: the GRAALL-2003 study. J Clin Oncol. 2009;27(6):911-918.

6. Toft N, Birgens H, Abrahamsson J, et al. Results of NOPHO ALL2008 treatment for patients aged 1-45 years with acute lymphoblastic leukemia. Leukemia. 2018;32(3):606-615.

7. Terwilliger T, Abdul-Hay M. Acute lymphoblastic leukemia: a comprehensive review and 2017 update. Blood Cancer J. 2017;7(6):e577.

8. Maury S, Chevret S, Thomas X, et al. Rituximab in B-Lineage Adult Acute Lymphoblastic Leukemia. N Engl J Med. 2016;375(11): 1044-1053.

9. Farhadfar N, Litzow MR. New monoclonal antibodies for the treatment of acute lymphoblastic leukemia. Leuk Res. 2016;49:13-21.

10. Hoelzer D. Targeted therapy with monoclonal antibodies in acute lymphoblastic leukemia. Curr Opin Oncol. 2013;25(6):701-706.

11. Jabbour E, O'Brien S, Ravandi F, Kantarjian H. Monoclonal antibodies in acute lymphoblastic leukemia. Blood. 2015;125(26): 4010-4016.

12. U.S. Food and Drug Administration (2017, August 17). FDA approves inotuzumab ozogamicin for relapsed or refractory B-cell precursor ALL [Press Release] Available from: https://www.fda.gov/Drugs/ InformationOnDrugs/ApprovedDrugs/ucm572133.htm. 2017. Accessed May 10, 2018.
13. Pfizer 2017 (June 30). BESPONSA $®$ Approved in the EU for Adult Patients with Relapsed or Refractory B-cell Precursor Acute Lymphoblastic Leukemia [Press Release]. Retrieved from http://press.pfizer. com/press-release/besponsa-approved-eu-adult-patients-relapsed-orrefractory-b-cell-precursor-acute-lymp. Accessed May 10, 2018.

14. Tedder TF, Tuscano J, Sato S, Kehrl JH, Cd KJH. CD22, a B lymphocyte-specific adhesion molecule that regulates antigen receptor signaling. Annu Rev Immunol. 1997;15:481-504.

15. Piccaluga PP, Arpinati M, Candoni A, et al. Surface antigens analysis reveals significant expression of candidate targets for immunotherapy in adult acute lymphoid leukemia. Leuk Lymphoma. 2011;52(2): 325-327.

16. Dijoseph JF, Armellino DC, Boghaert ER, et al. Antibody-targeted chemotherapy with CMC-544: a CD22-targeted immunoconjugate of calicheamicin for the treatment of B-lymphoid malignancies. Blood. 2004; 103(5):1807-1814.

17. Hinman LM, Hamann PR, Wallace R, Menendez AT, Durr FE, Upeslacis J. Preparation and characterization of monoclonal antibody conjugates of the calicheamicins: a novel and potent family of antitumor antibiotics. Cancer Res. 1993;53(14):3336-3342.

18. Shor B, Gerber HP, Sapra P. Preclinical and clinical development of inotuzumab-ozogamicin in hematological malignancies. Mol Immunol. 2015;67(2 Pt A):107-116.

19. George B, Kantarjian H, Jabbour E, Jain N. Role of inotuzumab ozogamicin in the treatment of relapsed/refractory acute lymphoblastic leukemia. Immunotherapy. 2016;8(2):135-143.

20. Advani A, Coiffier B, Czuczman MS, et al. Safety, pharmacokinetics, and preliminary clinical activity of inotuzumab ozogamicin, a novel immunoconjugate for the treatment of B-cell non-Hodgkin's lymphoma: results of a phase I study. J Clin Oncol. 2010;28(12):2085-2093.

21. Ogura M, Hatake K, Ando K, et al. Phase I study of anti-CD22 immunoconjugate inotuzumab ozogamicin plus rituximab in relapsed/refractory B-cell non-Hodgkin lymphoma. Cancer Sci. 2012;103(5):933-938.

22. Bassan R, Hoelzer D. Modern therapy of acute lymphoblastic leukemia. J Clin Oncol. 2011;29(5):532-543.

23. Dijoseph JF, Dougher MM, Evans DY, Zhou BB, Damle NK. Preclinical anti-tumor activity of antibody-targeted chemotherapy with CMC544 (inotuzumab ozogamicin), a CD22-specific immunoconjugate of calicheamicin, compared with non-targeted combination chemotherapy with CVP or CHOP. Cancer Chemother Pharmacol. 2011;67(4): 741-749.

24. de Vries JF, Zwaan CM, de Bie M, et al. The novel calicheamicinconjugated CD22 antibody inotuzumab ozogamicin (CMC-544) effectively kills primary pediatric acute lymphoblastic leukemia cells. Leukemia. 2012;26(2):255-264.

25. Dijoseph JF, Dougher MM, Armellino DC, Evans DY, Damle NK. Therapeutic potential of CD22-specific antibody-targeted chemotherapy using inotuzumab ozogamicin (CMC-544) for the treatment of acute lymphoblastic leukemia. Leukemia. 2007;21(11):2240-2245.

26. Kantarjian H, Thomas D, Jorgensen J, et al. Inotuzumab ozogamicin, an anti-CD22-calecheamicin conjugate, for refractory and relapsed acute lymphocytic leukaemia: a phase 2 study. Lancet Oncol. 2012; 13(4):403-411.

27. Kantarjian H, Thomas D, Jorgensen J, et al. Results of inotuzumab ozogamicin, a CD22 monoclonal antibody, in refractory and relapsed acute lymphocytic leukemia. Cancer. 2013;119(15):2728-2736.

28. Deangelo DJ, Stock W, Stein AS, et al. Inotuzumab ozogamicin in adults with relapsed or refractory CD22-positive acute lymphoblastic leukemia: a phase 1/2 study. Blood Adv. 2017;1(15):1167-1180.

29. Kantarjian HM, Deangelo DJ, Stelljes M, et al. Inotuzumab Ozogamicin versus Standard Therapy for Acute Lymphoblastic Leukemia. N Engl J Med Overseas Ed. 2016;375(8):740-753.

30. Jabbour EJ, Deangelo DJ, Stelljes M, et al. Efficacy and safety analysis by age cohort of inotuzumab ozogamicin in patients with relapsed or refractory acute lymphoblastic leukemia enrolled in INO-VATE. Cancer. 2018;124(8):1722-1732. 
31. Jabbour E, Ravandi F, Kebriaei P, et al. Salvage chemoimmunotherapy with inotuzumab ozogamicin combined with mini-hyper-CVD for patients with relapsed or refractory Philadelphia Chromosome-Negative Acute Lymphoblastic Leukemia. JAMA Oncol. 2018;4(2):230-234.

32. Ogura M, Tobinai K, Hatake K, et al. Phase I study of inotuzumab ozogamicin (CMC-544) in Japanese patients with follicular lymphoma pretreated with rituximab-based therapy. Cancer Sci. 2010;101(8): $1840-1845$.

33. Ogura M, Hatake K, Ando K, et al. Phase I study of anti-CD22 immunoconjugate inotuzumab ozogamicin plus rituximab in relapsed/refractory B-cell non-Hodgkin lymphoma. Cancer Sci. 2012;103(5):933-938.

34. Fayad L, Offner F, Smith MR, et al. Safety and clinical activity of a combination therapy comprising two antibody-based targeting agents for the treatment of non-Hodgkin lymphoma: results of a phase I/II study evaluating the immunoconjugate inotuzumab ozogamicin with rituximab. J Clin Oncol. 2013;31(5):573-583.

35. Dang NH, Ogura M, Castaigne S, et al. Randomized, phase 3 trial of inotuzumab ozogamicin plus rituximab versus chemotherapy plus rituximab for relapsed/refractory aggressive B-cell non-Hodgkin lymphoma. Br J Haematol. 2017;28.

36. Kantarjian HM, Deangelo DJ, Advani AS, et al. Hepatic adverse event profile of inotuzumab ozogamicin in adult patients with relapsed or refractory acute lymphoblastic leukaemia: results from the open-label, randomised, phase 3 INO-VATE study. Lancet Haematol. 2017; 4(8):e387-e398.

37. Mcdonald GB, et al. Veno-occlusive Disease of the Liver and Multiorgan Failure after Bone Marrow Transplantation: A Cohort Study of 355 Patients. Ann Intern Med. 1993;118(4):255.

38. Mcdonald GB, Sharma P, Matthews DE, Shulman HM, Thomas ED. Venocclusive disease of the liver after bone marrow transplantation: diagnosis, incidence, and predisposing factors. Hepatology. 1984;4(1): $116-122$.
39. Jones RJ, Lee KSK, Beschorner WE, et al. Venoocclusive disease of the liver following bone marrow transplantation. Transplantation. 1987; 44(6):778-783.

40. Palomo M, Diaz-Ricart M, Rovira M, Escolar G, Carreras E. Defibrotide Prevents the Activation of Macrovascular and Microvascular Endothelia Caused by Soluble Factors Released to Blood by Autologous Hematopoietic Stem Cell Transplantation. Biology of Blood and Marrow Transplantation. 2011;17(4):497-506.

41. Pescador R, Capuzzi L, Mantovani M, Fulgenzi A, Ferrero ME. Defibrotide: Properties and clinical use of an old/new drug. Vascul Pharmacol. 2013;59(1-2):1-10.

42. Richardson PG, Riches ML, Kernan NA, et al. Phase 3 trial of defibrotide for the treatment of severe veno-occlusive disease and multi-organ failure. Blood. 2016;127(13):1656-1665.

43. Kebriaei P, Cutler C, de Lima M, et al. Management of important adverse events associated with inotuzumab ozogamicin: expert panel review. Bone Marrow Transplant. 2018;53(4):449-456.

44. Kantarjian H, Stein A, Gökbuget N, et al. Blinatumomab versus Chemotherapy for Advanced Acute Lymphoblastic Leukemia. N Engl J Med Overseas Ed. 2017;376(9):836-847.

45. Park JH, Rivière I, Gonen M, et al. Long-Term Follow-up of CD19 CAR Therapy in Acute Lymphoblastic Leukemia. NEngl J Med Overseas Ed. 2018;378(5):449-459.

46. Mullard A. FDA approves first CAR T therapy. Nat Rev Drug Discov. 2017;16(10):669.

47. Reuters Staff. FDA approves Pfizer's drug for rare blood cancer; 2017, August 17. Available from: https://in.reuters.com/article/us-pfizer-fdaleukemia-idINKCN1AX2A0. Accessed July 7, 2018
Drug Design, Development and Therapy

\section{Publish your work in this journal}

Drug Design, Development and Therapy is an international, peerreviewed open-access journal that spans the spectrum of drug design and development through to clinical applications. Clinical outcomes, patient safety, and programs for the development and effective, safe, and sustained use of medicines are the features of the journal, which

\section{Dovepress}

has also been accepted for indexing on PubMed Central. The manuscript management system is completely online and includes a very quick and fair peer-review system, which is all easy to use. Visit http://www.dovepress.com/testimonials.php to read real quotes from published authors. 